\title{
Record-level Quantum Efficiency from a High Polarization Strained GaAs/GaAsP Superlattice Photocathode with Distributed Bragg Reflector
}

\author{
Wei Liu, ${ }^{1,2,3)}$ Yiqiao Chen, ${ }^{4)}$ Wentao Lu, ${ }^{4)}$ Aaron Moy, ${ }^{4)}$ Matt Poelker, ${ }^{3)}$ Marcy \\ Stutzman, ${ }^{3)}$ and Shukui Zhang ${ }^{3)}$ \\ ${ }^{1}$ Institute of Modern physics, Chinese Academy of Sciences, 509 Nanchang Rd., Lanzhou, 730000, China \\ ${ }^{2}$ University of Chinese Academy of Sciences, 19A Yuquan Rd., Beijing, 100049, China \\ ${ }^{3}$ Thomas Jefferson National Accelerator facility, 12000 Jefferson Avenue, Newport News, Virginia, 23606, \\ USA
}

${ }^{4}$ SVT Associates, Inc. 7620 Executive Dr., Eden Prairie, Minnesota, 55344, USA

Photocathodes that provide high electron-spin polarization (ESP) and high quantum efficiency (QE) can significantly enhance the physics capabilities of electron accelerators. We report record-level QE from a high-polarization strained GaAs/GaAsP superlattice photocathode fabricated with a Distributed Bragg Reflector (DBR). The DBR photocathode technique enhances the absorption of incident laser light thereby enhancing $\mathrm{QE}$, but as literature suggests, it is very challenging to optimize all of the parameters associated with the fabrication of complicated photocathode structures composed of many distinct layers. Past reports of DBR photocathodes describe high polarization but typically $\mathrm{QE}$ of only $\sim 1 \%$, which is comparable to $\mathrm{QE}$ of high polarization photocathodes grown without a DBR structure. This work describes a strained GaAs/GaAsP superlattice DBR photocathode exhibiting high polarization of $84 \%$ and significantly enhanced QE of $6.4 \%$.

Spin-polarized electron sources play a critical role in nuclear and high energy physics research, where the spin of the electron is used to study nuclear structure, the dynamics of strong interactions, electro-weak nuclear physics, including parity-violation, and physics beyond the Standard Model. ${ }^{1}$ Inexpensive bulk GaAs provides very high quantum efficiency (QE) but electron-spin polarization (ESP) cannot exceed 50\% due to the heavy-hole, light-hole degeneracy of the ${ }^{2} \mathrm{p}_{3 / 2}$ valence band state. ${ }^{2}$ Photocathodes grown on a crystal structure with a different lattice constant can provide beam polarization exceeding $50 \%$ because of induced uniaxial strain that eliminates the degeneracy of the valence band. ${ }^{3,4}$ Throughout the 1990s, single-strained-layer GaAs/GaAsP photocathodes providing polarization $75 \%-80 \%$ and maximum $\mathrm{QE} \sim 0.3 \%{ }^{3,5,6}$ were used at electron accelerators worldwide ${ }^{7-13}$, but beam delivery using single-strained-layer photocathodes made apparent the delicate competing balance between maintaining the required degree of strain, and growing a layer thick enough to provide sufficient QE. In the 
following decade, strained superlattice structures consisting of very thin quantum-well active layers and alternating lattice-mismatched barrier layers were developed. ${ }^{14}$ The superlattice structure maintained the required degree of strain to produce high polarization and also provided sufficient active layer thickness to obtain higher QE. The strained $\mathrm{GaAs} / \mathrm{GaAsP}_{0.36}$ superlattice structure reported in ref. 15 provided QE of $1.2 \%$ with polarization of $86 \%$ and is available commercially ${ }^{16}$. Strained superlattice photocathodes fabricated at other facilities have also demonstrated very high polarization but no higher QE. ${ }^{17-20}$

One of the proposed Electron Ion Collider (EIC) designs, eRHIC ${ }^{21}$, requires milliamperes of polarized electron beam representing a factor of $\sim 250$ beyond today's state-of-the-art. A photocathode with a QE of only $1 \%$ would require $\sim 8 \mathrm{~W}$ of laser light (with RF structure) to generate the desired $50 \mathrm{~mA}$ average beam current and even more light when $\mathrm{QE}$ decays during operation due to ion-bombardment ${ }^{22}$. In the standard strained superlattice design, most of the incident laser light simply heats the photocathode instead of promoting electron ejection, which can degrade QE due to evaporation of the chemicals used to reduce the surface work function. ${ }^{23}$ Methods to cool the photocathode during beam delivery are complicated because the photocathode floats at high voltage. Developing photocathodes with enhanced QE could simplify the photogun design, reduce the drive-laser power requirements and prolong the effective operating lifetime of the photogun.

A photocathode with a distributed Bragg reflector (DBR) was first proposed in $1993^{24}$ and reported in following years $^{25,26}$ as a means to enhance photocathode QE. The DBR serves to create a Fabry-Perot cavity formed by the front surface of photocathode and a DBR region existing beneath the superlattice active layer. Instead of a single pass in the standard design, laser light of a particular wavelength reflects repeatedly within the Fabry-Perot cavity which increases the beneficial absorption of the incident photons, and in principle, leads to enhanced QE. In our previous work $^{27}$, the enhanced QE of a strained GaAs/GaAsP superlattice photocathode with DBR was $\sim 1.2 \%$ with polarization near 90\%. Relatively low QE was attributed to weak resonant absorption of incident laser light and a mismatch between the wavelength of peak absorption and peak polarization. It was clear that the wavelength of peak reflectivity of the DBR was very sensitive to the refractive indices and thickness of each DBR layer constituent, and that the peak of resonant absorption was very sensitive to the thickness of the photocathode, especially the spacer layer between the DBR and the superlattice photocathode structures. Accurate control of the thickness of each layer and of the composition of each constituent chemical within the photocathode structure was challenging. In this study, a deeper appreciation for the global challenges of photocathode fabrication, together with improved modeling and fabrication techniques, allowed the fabrication of a strained GaAs/GaAsP superlattice photocathode with DBR (GaAsP/AlAsP) exhibiting significantly improved performance. The photocathode provided $84 \%$ polarization and $\mathrm{QE}$ of $6.4 \%$, which is the highest $\mathrm{QE}$ of any reported "high polarization" photocathode. Moreover, peak polarization and QE were obtained at $776 \mathrm{~nm}$, a wavelength compatible with CEBAF photogun drive lasers and readily available fiber-telecommunications hardware.

This DBR study benefited from past work that resulted in the fabrication of the strained GaAs/GaAsP superlattice photocathode described in ref. 15, which represents the basis for polarization and QE comparison. See 
ref. 15 for a complete description of the design criteria and optimization of this photocathode structure. A schematic layout of the strained GaAs/GaAsP superlattice photocathodes with and without DBR structure is shown in Figure 1.

\begin{tabular}{|c|c|c|}
\hline GaAs & $5 \mathrm{~nm}$ & $\mathrm{p}=5 \cdot 10^{19} \mathrm{~cm}^{-3}$ \\
\hline $\begin{array}{c}\text { GaAs/GaAsP } \\
\text { SL }\end{array}$ & $\begin{array}{c}(3.8 / 2.8 \mathrm{~nm}) \\
\times 14\end{array}$ & $p=5 \cdot 10^{17} \mathrm{~cm}^{-3}$ \\
\hline GaAsP $_{0.35}$ & $2750 \mathrm{~nm}$ & $p=5 \cdot 10^{18} \mathrm{~cm}^{-3}$ \\
\hline $\begin{array}{c}\text { Graded GaAsP } P_{x} \\
\quad(x=0 \sim 0.35)\end{array}$ & $5000 \mathrm{~nm}$ & $p=5 \cdot 10^{18} \mathrm{~cm}^{-3}$ \\
\hline GaAs buffer & $200 \mathrm{~nm}$ & $\mathrm{p}=2 \cdot 10^{18} \mathrm{~cm}^{-3}$ \\
\hline
\end{tabular}

\begin{tabular}{|c|c|c|}
\hline GaAs & $5 \mathrm{~nm}$ & $\mathrm{p}=5 \cdot 10^{19} \mathrm{~cm}^{-3}$ \\
\hline $\begin{array}{c}\text { GaAs/GaAsP } \\
\text { SL }\end{array}$ & $\begin{array}{c}(3.8 / 2.8 \mathrm{~nm}) \\
\times 14\end{array}$ & $\mathrm{p}=5 \cdot 10^{17} \mathrm{~cm}^{-3}$ \\
\hline $\mathrm{GaAsP}_{0.35}$ & $750 \mathrm{~nm}$ & $p=5 \cdot 10^{18} \mathrm{~cm}^{-3}$ \\
\hline $\begin{array}{c}\mathrm{GaAsP}_{0.35} / \mathrm{AlAsP}_{0.4} \\
\text { DBR }\end{array}$ & $\begin{array}{c}(54 / 64 \mathrm{~nm}) \\
\quad \times 12\end{array}$ & $\mathrm{p}=5 \cdot 10^{18} \mathrm{~cm}^{-3}$ \\
\hline $\mathrm{GaAsP}_{0.35}$ & $2000 \mathrm{~nm}$ & $\mathrm{p}=5 \cdot 10^{18} \mathrm{~cm}^{-3}$ \\
\hline $\begin{array}{l}\text { Graded GaAsP }_{x} \\
\quad(x=0 \sim 0.35)\end{array}$ & $5000 \mathrm{~nm}$ & $\mathrm{p}=5 \cdot 10^{18} \mathrm{~cm}^{-3}$ \\
\hline GaAs buffer & $200 \mathrm{~nm}$ & $\mathrm{p}=2 \cdot 10^{18} \mathrm{~cm}^{-3}$ \\
\hline
\end{tabular}

FIG. 1. Schematic structure of the photocathodes: without DBR (left) and with DBR (right).

The DBR is composed of multiple layers of alternating high and low index of refraction. A natural choice for high index of refraction layer was GaAsP which provides a good lattice match to the graded buffer layer. Though a challenge for growth, we chose $\mathrm{AlAs}_{1-\mathrm{y}} \mathrm{P}_{\mathrm{y}}$ for the low index of refraction layer for maximum refractive index contrast and minimum DBR thickness. The thickness of each layer follows the quarter wavelength condition: ${ }^{24} n_{H}\left(\lambda_{D B R}\right) d_{H}=\frac{\lambda_{D B R}}{4}=n_{L}\left(\lambda_{D B R}\right) d_{L}$. The bandwidth for which the DBR exhibits high reflectivity is given by the formula:

$$
\Delta \lambda_{D B R}=\frac{4 \lambda_{D B R}}{\pi} \sin ^{-1}\left(\frac{n_{H}\left(\lambda_{D B R}\right)-n_{L}\left(\lambda_{D B R}\right)}{n_{H}\left(\lambda_{D B R}\right)+n_{L}\left(\lambda_{D B R}\right)}\right)
$$

The Fabry-Perot cavity is formed by the vacuum/GaAs cap layer interface and the DBR. Proper thickness of a GaAsP spacer layer between GaAs/GaAsP superlattice and the DBR ensures the maximum absorption in superlattice active layer coincide with the central reflective wavelength of the DBR. To design the structure, the refractive index of these layers can be estimated using the simplified interband transition model as a function of photon energy ${ }^{28}$ :

$$
n(E)^{2}=A\left[f\left(X_{0}\right)+\frac{1}{2}\left(\frac{E_{0}}{E_{0}+\Delta_{0}}\right)^{\frac{3}{2}} f\left(X_{s o}\right)\right]+B
$$

with $f(X)=X^{-2}(2-\sqrt{1+X}-\sqrt{1-X}), X_{0}=\frac{E}{E_{0}}$, and $X_{s o}=\frac{E}{E_{0}+\Delta_{0}}$. Here, $A$ represents the strength parameter of the $E /\left(E_{0}+\Delta_{0}\right)$ terms and $B$ represents the nondispersive contribution arising from the higher-lying band gap terms $\left(E_{1}, E_{1}+\Delta_{1}, E_{2}\right.$, etc. $)$ for each layer. The quantities $E_{0}$ and $\Delta_{0}$ are the band-gap energy and spin-orbit splitting, respectively, given by Vegard's Law for $\mathrm{GaAs}_{1-x} P_{x}^{29,30}$. 
The desired wavelength of peak reflectivity was $\lambda_{D B R} \sim 780 \mathrm{~nm}$, compatible with CEBAF drive lasers. Setting the phosphorus fractions in the $\operatorname{GaAs}_{1-\mathrm{x}} \mathrm{P}_{\mathrm{x}}$ and $\mathrm{AlAs}_{1-\mathrm{y}} \mathrm{P}_{\mathrm{y}}$ layers to 0.35 and 0.4 , respectively, provided refractive indices of $n_{H}(780 \mathrm{~nm})=3.4506$ and $n_{L}(780 \mathrm{~nm})=2.9443$. From these parameters, the design values for the thickness of the $\mathrm{GaAsP}_{0.35}$ and $\mathrm{AlAsP}_{0.4}$ layers were $56.5 \mathrm{~nm}$ and $66.5 \mathrm{~nm}$, respectively. Actual layer thicknesses were very close to these design values, $54 \mathrm{~nm}$ and $64 \mathrm{~nm}$, which set the wavelength of peak reflectivity to $\lambda_{D B R}$ of $755 \mathrm{~nm}$, and the reflectivity bandwidth $\Delta \lambda_{D B R} \sim 79 \mathrm{~nm}$. This implies the DBR can achieve a high reflectivity $>90 \%$ for wavelengths between 715 and $795 \mathrm{~nm}$.

The transfer matrix method ${ }^{32,33}$ was used to calculate the reflection, transmission and absorption of incident light through the complete multilayer thin-film structure including the GaAs substrate. Calculated values of surface reflectivity (R), transmittance (T) into the GaAs substrate, absorption (A), and the absorption enhancement factor as a function of wavelength, are shown in Figure 2. There are two resonant absorption peaks within the optical cavity for the DBR photocathode at wavelengths $726 \mathrm{~nm}$ and $776 \mathrm{~nm}$. Absorption by the substrate is not plotted as this process does not contribute to the polarized electron emission. The resonant peak at $776 \mathrm{~nm}$ is just $4 \mathrm{~nm}$ from the desired value $780 \mathrm{~nm}$. At $776 \mathrm{~nm}$, the absorption is $21.03 \%$, surface reflectivity is $50.14 \%$, and transmittance into GaAs substrate is $28.83 \%$ for the DBR photocathode, corresponding to maximum electron spin polarization. The full-width half-maximum of the Fabry-Perot resonance is about $10 \mathrm{~nm}$ at $776 \mathrm{~nm}$. The absorption enhancement factor is 7.4 at this wavelength.

To estimate the quantum efficiency of photocathode, one can solve the one-dimensional diffusion equations, based on Spicer's three-step model: ${ }^{34,35}$

$$
Q E(\lambda)=\frac{P_{L} F_{L} A}{1+\frac{1}{\alpha_{\lambda} L_{L}}}+\frac{P_{\Gamma} \exp \left[k\left(-\frac{1}{1.42}-\frac{\lambda}{1240}\right)\right] A}{1+\frac{1}{\alpha_{\lambda} L_{\Gamma}}}\left[F_{\Gamma}+\frac{F_{L} L_{\Gamma}}{\alpha_{\lambda} F_{L}\left(L_{\Gamma}+L_{L}\right)\left(1+\frac{1}{\alpha_{\lambda} L_{L}}\right)}\right]
$$

where $P_{\Gamma}$ and $P_{\mathrm{L}}$ are the surface electron escape probabilities for the $\Gamma$ and $L$ minima, respectively, which are independent of the incident photon energy, $F_{\Gamma}$ is the remaining fraction of excited electrons, $F_{\mathrm{L}}$ is the fraction of electrons that is excited to energies greater than the $L$ minima, $A$ is the absorption of photocathode, $L_{\Gamma}$ and $L_{L}$ are the electron diffusion length for the $\Gamma$ and $\mathrm{L}$ minima, respectively, $\alpha_{\lambda}$ is the absorption coefficient of the photocathode, and $k$ is a coefficient $(k \geq 0)$. The calculated QE for the DBR photocathode, together with the QE enhancement factor as a function of wavelength, are shown in Figure 2. 


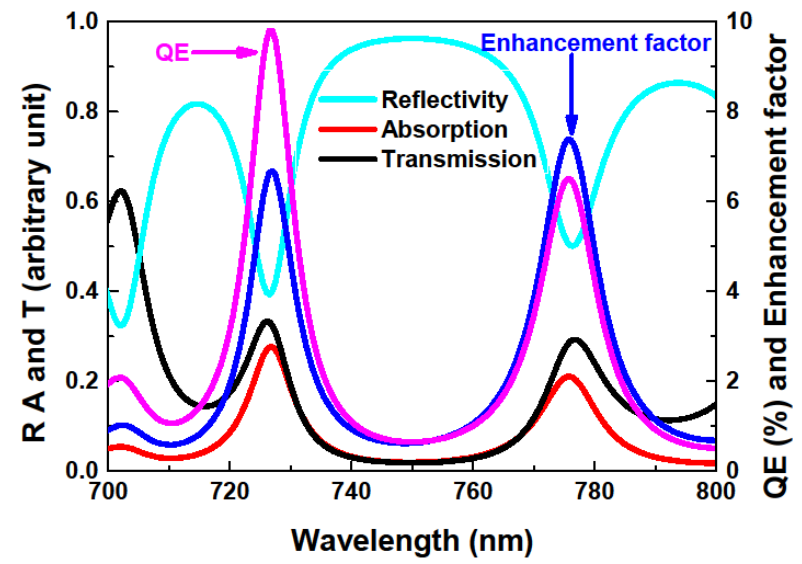

Fig. 2. Calculated values of absorption, reflectivity and transmittance of the DBR photocathode, as a function of wavelength. Also shown, calculated QE and the QE enhancement factor compared to the photocathode without the DBR.

All photocathodes were grown in a solid-source molecular beam epitaxy (SSMBE) system equipped with As and P crackers on a (100) p-type GaAs substrate, upon which a $200 \mathrm{~nm}$ Be-doped $\left(2 \times 10^{18} \mathrm{~cm}^{-3}\right) \mathrm{GaAs}$ buffer layer was deposited. The 5 - $\mu \mathrm{m}$-thick Be-doped graded $\mathrm{GaAsP}_{\mathrm{x}}$ layer was then grown with an increasing $\mathrm{P}$ composition from $\mathrm{x}=0$ to 0.35 , followed by a $2-\mu \mathrm{m}$-thick $\mathrm{GaAs}_{0.65} \mathrm{P}_{0.35}$ layer, in order to produce a strain-relaxed $\mathrm{GaAs}_{0.65} \mathrm{P}_{0.35}$ layer compatible with the superlattice active layer. The DBR structure consisting of 12-pair of $\mathrm{GaAs}_{0.65} \mathrm{P}_{0.35}$ (54 $\mathrm{nm}) / \mathrm{AlAs}_{0.6} \mathrm{P}_{0.4}(64 \mathrm{~nm})$ was subsequently grown on top of this layer. In order to create an effective Fabry-Perot resonant cavity for the desired wavelength near $780 \mathrm{~nm}$, a 750 -nm-thick $\mathrm{GaAs}_{0.65} \mathrm{P}_{0.35}$ layer was grown on top of DBR acting as a spacer. Both GaAs $0.65 \mathrm{P}_{0.35}$ and DBR layers were Be-doped at $5 \times 10^{18} \mathrm{~cm}^{-3}$ to promote high electrical conductivity. The p-type $\left(5 \times 10^{17} \mathrm{~cm}^{-3}\right)$ GaAs $(3.8 \mathrm{~nm}) / \mathrm{GaAs}_{0.65} \mathrm{P}_{0.35}(2.8 \mathrm{~nm})$ superlattice layer was positioned between the $\mathrm{GaAs}_{0.65} \mathrm{P}_{0.35}$ spacer and the 5-nm-thick GaAs cap layer doped at $\mathrm{p}=5 \times 10^{19} \mathrm{~cm}^{-3}$. The only difference between the DBR photocathode and the non-DBR photocathode was the 12-pairs of $\mathrm{GaAs}_{0.65} \mathrm{P}_{0.35}$ (54 $\mathrm{nm}) / \mathrm{AlAs}_{0.6} \mathrm{P}_{0.4}(64 \mathrm{~nm})$ layers.

The growth of $\mathrm{GaAs}_{0.65} \mathrm{P}_{0.35} / \mathrm{AlAs}_{0.6} \mathrm{P}_{0.4}$ DBR structure was very challenging due to the large differences in growth temperatures and the V/III flux ratios for the $\mathrm{GaAs}_{0.65} \mathrm{P}_{0.35}$ and $\mathrm{AlAs}_{0.6} \mathrm{P}_{0.4}$ layers. The optimal growth temperatures for GaAs0.65 $\mathrm{P}_{0.35}$ and $\mathrm{AlAs} 0.6 \mathrm{P}_{0.4}$ were $660-700{ }^{\circ} \mathrm{C}$ and $700-750{ }^{\circ} \mathrm{C}$, respectively, as determined by surface temperature measurement and reflective high energy electron diffraction (RHEED) images during MBE growth. Thicknesses and composition were verified using $\mathrm{x}$-ray diffraction measurements and $\mathrm{x}$-ray simulation models. It was very difficult to accurately control the growth rate of $\mathrm{GaAs}_{0.65} \mathrm{P}_{0.35}$ at high temperatures, especially approaching $700{ }^{\circ} \mathrm{C}$, because the re-evaporation rate of $\mathrm{Ga}$ atoms from the sample surface increases rapidly with increasing growth temperatures above $660{ }^{\circ} \mathrm{C}$, resulting in inconsistent control of the layer thickness. Additionally, maintaining the As/P fraction in $\mathrm{GaAs}_{0.65} \mathrm{P}_{0.35}$ layers is difficult when grown below $650{ }^{\circ} \mathrm{C}$, as the As/P fraction is very sensitive at low growth temperatures. ${ }^{36,37}$ For these reasons the thick graded composition GaAsP was grown at $660{ }^{\circ} \mathrm{C}$. However, the material quality of $\mathrm{AlAs}_{0.6} \mathrm{P}_{0.4}$ layer was poor when grown at this temperature. Therefore, the 
growth temperature of $\mathrm{GaAs}_{0.65} \mathrm{P}_{0.35} / \mathrm{AlAs}_{0.6} \mathrm{P}_{0.4} \mathrm{DBR}$ had to be kept at $700{ }^{\circ} \mathrm{C}$ as measured by an SVT AccuTemp process monitor using a two-color infrared pyrometer. The large difference in V/III flux ratio during the growth between $\mathrm{GaAs}_{0.65} \mathrm{P}_{0.35}$ and $\mathrm{AlAs}_{0.6} \mathrm{P}_{0.4}$ added extra difficulty to achieving high quality DBR layers. The $\mathrm{V} / \mathrm{III}$ ratio for $\mathrm{GaAs}_{0.65} \mathrm{P}_{0.35}$ is around 50, whereas the ratio is only around 3 for $\mathrm{AlAs}_{0.6} \mathrm{P}_{0.4}$. Numerous growth-calibration and material-characterization runs were required to develop suitable substrate temperature and source-switching recipes to enable the fabrication of high quality $\mathrm{GaAs}_{0.65} \mathrm{P}_{0.35} / \mathrm{AlAs} 0.6 \mathrm{P}_{0.4} \mathrm{DBR}$ and strained-superlattice structures grown by SSMBE.

Photocathode samples were evaluated using a low-voltage retarding-field Mott polarmeter ${ }^{38}$. Samples were attached to sample holders and installed within the vacuum chamber which was baked at $250{ }^{\circ} \mathrm{C}$ for 36 hours, and allowed to cool to room temperature. Photocathodes were then heated to $480{ }^{\circ} \mathrm{C}$ to remove the As cap and cooled to room temperature under typical vacuum pressure $\sim 10^{-11}$ Torr. A negative electron affinity condition was obtained using the standard yo-yo activation procedure with cesium and $\mathrm{NF}_{3} .{ }^{39}$ A broadly tunable super-continuum laser (NKT Photonics, SuperK) provided milli-Watts of output power over the wavelength range from 400 to $800 \mathrm{~nm}$. Optical waveplates (quarter and halfwave) were used to generate left and right circularly polarized light required to obtain spin polarized electrons.

Measured values of QE and polarization as a function of wavelength, for photocathodes with and without DBR, are shown in Figure 3a. For the non-DBR photocathode, results are consistent with past work ${ }^{15}$ indicating peak polarization of $90 \%$ and $\mathrm{QE}$ of $0.89 \%$ at a wavelength $780 \mathrm{~nm}$. In stark contrast, the QE of the DBR photocathode shows the telltale prominent oscillatory behavior indicative of resonant absorption. Measured reflectivity and QE enhancement (i.e., the ratio of QE values of photocathodes with and without DBR) are shown in Figure $3 \mathrm{~b}$. The two dips in the reflectivity spectrum are clearly correlated with wavelength locations of QE maxima. At the wavelength of interest, $776 \mathrm{~nm}$, the $\mathrm{QE}$ was $6.4 \%$ and polarization $\sim 84 \%$. The measured QE enhancement of $\sim 7.2$ is very close to the predicted value of $\sim 7.4$. It should be noted that QE enhancement is a somewhat arbitrary metric dependent on when measurements were made following photocathode activation and subject to non-trivial variations in $\mathrm{QE}$ associated with vacuum conditions that influence photocathode lifetime. 


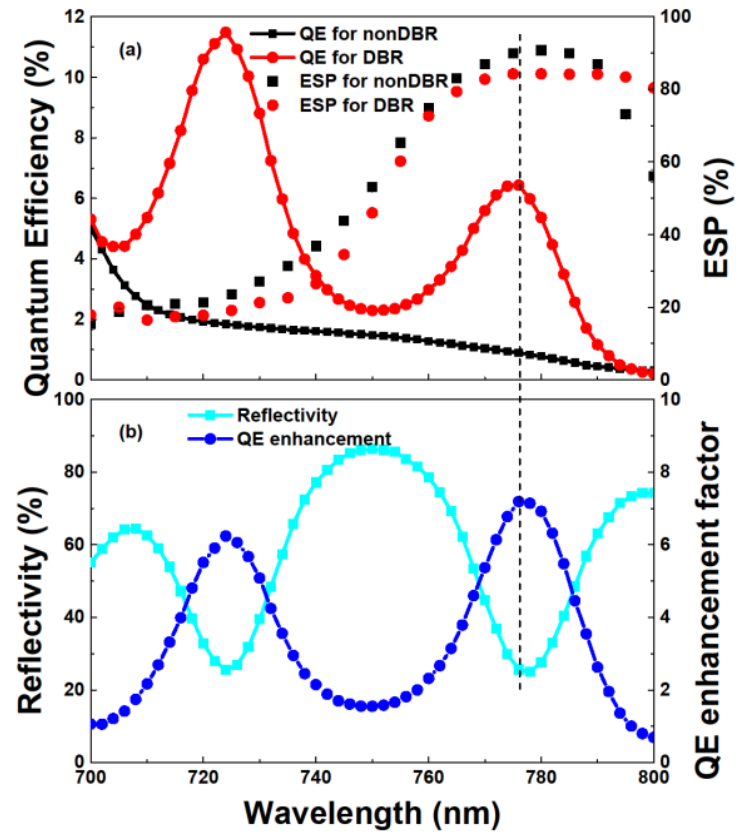

Fig. 3. (a) The QE and electron-spin polarization (ESP) for the strained GaAs/GaAsP superlattice photocathodes with and without DBR as a function of the wavelength; (b) Reflectivity and QE enhancement factor of DBR photocathode as a function of the wavelength. The dash line indicates the resonant position.

These results are compared to results from other photocathodes listed in Table 1, showing QE, polarization and photocathode Figure of Merit $\left(P^{2} Q E\right)$. The Figure of Merit of our photocathode is a factor of four higher than the last highest value. In conclusion, a $\mathrm{QE}$ of $6.4 \%$ is the highest reported value of any "high polarization" photocathode. Precise control of the thickness of each layer and the composition of each constituent led to these improved results. Further optimization should lead to even higher QE, and polarization values approaching the best values from superlattice photocathodes without DBR structures.

Table 1. Figure of merit for polarized electron sources.

\begin{tabular}{|c|c|c|c|c|}
\hline Cathode & Ref. & $P(\%)$ & QE (\%) & $\begin{array}{c}\text { Figure of Merit } \\
\left(P^{2} Q E\right)\end{array}$ \\
\hline $\mathrm{GaAs}_{\mathrm{GaAsP}} \mathrm{G}_{0.36}$ (no DBR) & SLAC/SVT ${ }^{15}$ & 86 & 1.2 & 0.89 \\
\hline GaAs/GaAsP ${ }_{0.38}$ (no DBR) & Nagoya $^{20}$ & 92 & 1.6 & 1.35 \\
\hline $\begin{array}{c}\mathrm{Al}_{0.19} \mathrm{In}_{0.2} \mathrm{GaAs} / \mathrm{Al}_{0.4} \mathrm{GaAs} \\
\text { (with DBR) }\end{array}$ & St. Peterburg ${ }^{18}$ & 92 & 0.85 & 0.72 \\
\hline $\begin{array}{c}\text { GaAs/GaAsP } 0.35 \\
\text { (with DBR) }\end{array}$ & JLab/SVT & 84 & 6.4 & 4.52 \\
\hline
\end{tabular}

Authored by Jefferson Science Associates, LLC under U.S. DOE Contract No. DE-AC05-06OR23177. The U.S. Government retains a non-exclusive, paid-up, irrevocable, world-wide license to publish or reproduce this manuscript for U.S. Government purposes. SVT Associates was funded by the U. S. DOE's Office of Nuclear 
Physics SBIR program DE-SC0009516. W. Liu is currently conducting research at Jefferson Lab toward a PhD. degree.

${ }^{1}$ H. Montgomery, “Jefferson Lab: A Long Decade of Physics”, J. Phys.: Conf. Ser. 299 (2011) 011001

${ }^{2}$ D. T. Pierce, and F. Meier, "Photoemfssion of spin-polarized electrons from GaAs", Phys. Rev. B 13, 5484 (1976)

${ }^{3}$ T. Nakanishi, H Aoyagi, H. Horinaka, Y. Kamiya, T. Kato, S. Nakamura, T. Saka, and M. Tsubata, "Large enhancement of spin polarization observed by photoelectrons from the strained GaAs layer" Phys. Lett. A 158, $345(1991)$

${ }^{4}$ T. Maruyama, E. L. Garwin, R. Prepost, G. H. Zapalac, J. S. Smith, and J. D. Walker, “Observation of strainenhanced electron-spin polarization in photoemission from InGaAs”, Phys. Rev. Lett. 66, 2376 (1991)

${ }^{5}$ Bandwidth Semiconductor, LLC, 25 Sagamore Park Drive, Hudson, NH 03051, http://www.bandwidthsemi.com

${ }^{6}$ T. Maruyama, E. L. Garwin, R. Prepost, and G. H. Zapalac, "Electron-spin polarization in photoemission from strained GaAs Grown on GaAs ${ }_{1-x} P_{x} ”$, Phys. Rev. B 46, 4261 (1992)

${ }^{7}$ K. Aulenbacher, Ch. Nachtigall, H. G. Andresen, J. Bermuth, Th. Dombo, P. Drescher, H. Euteneuer, H. Fischer, D. v. Harrach, P. Hartmann, et al., "The MAMI source of polarized electrons", Nucl. Instrum. Meth. A, vol. 391, pp. 498-506, June 1997.

${ }^{8}$ G. D. Cates, V. W. Hughes, R. Michaels, H. R. Schaefer, T. J. Gay, M. S. Lubell, R. Wilson, G.W. Dodson, K. A. Dow, S. B. Kowalski, et al., "The Bates polarized electron source”, Nucl. Instrum. Meth. A, vol. 278, pp. 293-317, June 1989.

${ }^{9}$ E. Tsentalovich, D. Barkhuff, J. Chen, G. Dodson, M. Farkhondeh, W. Franklin, E. Ihloff, F. Kaertner, C. Tschalaer, B. Yang, T. Zwart, "Development of a polarized electron source for the MIT-Bates Linear Accelerator Center, Nucl. Instrum. Meth. A 582, 413-428 (2007)

${ }^{10}$ M. J. J. van den Putte, C. W. De Jager, S. G. Konstantinov, V. Ya. Korchagin, F. B.Kroes, E. P. vanLeeuwen, B. L. Militsyn, N. H. Papadakis, S. G. Popov, G. V. Serdobintsev, et al., "The polarized electron source at NIKHEF", in AIP Conf. Proc., vol. 421, 1997, pp. 260-269.

${ }^{11}$ W. Hillert, M. Govin, and B. Neff, "The $50 \mathrm{keV}$ inverted source of polarized electrons at ELSA”, in AIP Conf. Proc., vol. 570, 2000, pp. 961-964.

${ }^{12}$ C. K. Sinclair, P. A. Adderley, B. M. Dunham, J. C. Hansknecht, P. Hartmann, M. Poelker, J. S. Price, P. M. Rutt, W. J. Schneider, and M. Steigerwald, "Development of a high average current polarized electron source with long cathode operational lifetime", Phys. Rev. ST Accel. Beams, vol. 10, pp. 023501-1-023501-21, February 2007.

${ }^{13}$ R. Alley, H. Aoyagi , J. Clendenin, J. Frisch, C. Garden, E. Hoyt, R. Kirby, L. Klaisner, A. Kulikov, R. Miller, et al., "The Stanford linear accelerator polarized electron source", Nucl. Instrum. Meth. A, vol. 365, pp. 1-27, November 1995. 
${ }^{14}$ T. Omori, Y. Kurihara, Y. Takeuchi, M. Yoshioka, T. Nakanishi, S. Okumi, M. Tsubata, M. Tawada, K. Togawa, Y. Tanimoto, C. Takahashi, T. Baba, and M. Mizuta, "Highly polarized electron source using InGaAs-GaAs strained-layer superlattice”, Jpn. J. Appl. Phys. 33, 5676 (1994)

15 T. Maruyama, D. A. Luh, A. Brachmann, J. E. Clendenin, E. L. Garwin, S. Harvey, J. Jiang, R. E. Kirby, C. Y. Prescott, R. Prepost, and A. M. Moy, "Systematic study of polarized electron emission from strained GaAs/GaAsP superlattice photocathodes" Appl. Phys. Lett. 85, 2640 (2004)

${ }^{16} \mathrm{http}: / /$ www.svta.com/

${ }^{17}$ A. V. Subashiev, L. G. Gerchikov, Y. A. Mamaev, Y. P. Yashin, J. S. Roberts, D.-A. Luh, T. Maruyama, and J. E. Clendenin, "Strain-compensated AlInGaAs-GaAsP superlattices for highly polarized electron emission”, Appl. Phys. Lett. 86, 171911 (2005)

${ }^{18}$ Yu. A. Mamaev, L. G. Gerchikov, Yu. P. Yashin, D. A. Vasiliev, V. V. Kuzmichev, V. M. Ustinov, A. E. Zhukov, V. S. Mikhrin, and A. P. Vasiliev, “Optimized photocathode for spin-polarized electron sources”, Appl. Phys, Lett. 93, 081114 (2008)

${ }^{19}$ X. Jin, A. Mano, F. Ichihashi, N. Yamamoto, and Y. Takeda, "High-Performance Spin-Polarized Photocathodes Using a GaAs/GaAsP Strain-Compensated Superlattice”, Appl. Phys. Express 6, 015801 (2013)

${ }^{20}$ X. Jin, B. Ozdol, M. Yamamoto, A. Mano, N. Yamamoto, and Y. Takeda, "Effect of crystal quality on performance of spin-polarized photocathode”, Appl. Phys. Lett. 105, 203509 (2014)

${ }^{21}$ E. C. Aschenauer, M. D. Baker, A. Bazilevsky, K. Boyle, S. Belomestnykh, I. Ben-Zvi, S. Brooks, C. Brutus, T. Burton, S. Fazio, et al., “eRHIC design study: An electron-ion collider at BNL”, arXiv: 1409. 1633

${ }^{22}$ Wei Liu, Shukui Zhang, Marcy Stutzman, and Matt Poelker, “The effects of ion bombardment on bulk GaAs photocathodes with different surface-cleavage planes", Phys. Rev. Accel. Beam 19, 103402 (2016)

${ }^{23}$ S. Zhang, S. V. Benson, and C. H-Garcia, "Observation and measurement of temperature rise and distribution on GaAs photo-cathode wafer with a $532 \mathrm{~nm}$ drive laser and a thermal imaging camera”, Nucl. Instrum. Meth. A. $631,22(2011)$

${ }^{24}$ T. Saka, T. Kato, T. Nakanishi, M. Tsubata, K. Kishino, H. Horinaka, Y. Kamiya, S. Okumi, C. Takahashi, Y. Tanimoto, et al., "New-type photocathode for polarized electron source with Distributed Bragg Reflector”, Jpn. J. Appl. Phys. 32, 1837 (1993)

${ }^{25}$ J. C. Grobli, D. Oberli, and F. Meier, "Polarization resonances of optically spin-oriented photoelectrons emitted from strained semiconductor photocathodes”, Phys. Rev. Lett. 74, 2106 (1995)

${ }^{26}$ L. G. Gerchikov, K. Aulenbacher, J. E. Clendenin, V. V. Kuz'michev, Y. A. Mamaev, T. Maruyama, V. S. Mikhrin, J. S. Roberts, V. M. Ustinov, D. A. Vasilev, A. P. Vasiliev, Y. P. Yashin, and A. E. Zhukov, “Highly Effective Polarized Electron Sources Based on Strained Semiconductor Superlattice with Distributed Bragg Reflector”, AIP Conf. Proc. 980, 124 (2008) 
27 S. Zhang, M. L. Stutzman, M. Poelker, Y. Chen, and Aaron Moy, “Observation of significant quantum efficiency enhancement from a polarized photocathode with distributed brag reflector”, Proceedings of IPAC2015, Richmond, USA (2015)

${ }^{28}$ S. Adachi, "Properties of semiconductor alloys: group-IV, III-V and II-VI Semiconductors", (Wiley, 2009) P.238

${ }^{29}$ M. Levinshtein, S. Rumyantsev, M. Shur, "Handbook series on semiconductor parameters”, (World Scientific, 1996)

${ }^{30}$ I. Vurgaftman, J. R. Meyer, and L. R. Ram-Mohan, "Band parameters for III-V compound semiconductors and their alloys" J. Appl. Phys. 89, 5815 (2001)

${ }^{31}$ D. E. Aspnes, S. M. Kelso, R. A. Logan, and R. Bhat, “Optical properties of $\mathrm{Al}_{\mathrm{x}} \mathrm{Ga}_{1-\mathrm{x}} \mathrm{As}$ ”, J. Appl. Phys. 60,754 (1986)

${ }^{32}$ H. A. Macleod, “ Thin-film optical filters”, $4^{\text {th }}$ edition, (CRC Press,2010)

${ }^{33}$ P. Peumans, A. Yakimov, and S. R. Forrest, "Small molecular weight organic thin-film photodetectors and solar cells”, J. Appl. Phys. 93, 3693 (2003)

${ }^{34}$ J. Zou, B. Chang, H. Chen, and L. Liu, "Variation of quantum-yield curves for GaAs photocathodes under illumination”, J. Appl. Phys. 101, 033126 (2007)

35 J. Zou, B. Chang, Z. Yang, Y. Zhang, and J. Qiao, "Evolution of surface potential barrier for negative-electronaffinity GaAs photocathodes”, J. Appl. Phys. 105, 013714 (2009)

${ }^{36}$ B.W. Liang and C.W. Tu, "A kinetic model for As and P incorporation behaviors in GaAsP grown by gas-source molecular beam epitaxy” J. Appl. Phys. 74, 255 (1993)

${ }^{37}$ Stephanie Tomasulo, John Simon, Paul J. Simmonds, Jonathan Biagiotti, and Minjoo L. Lee, "Molecular beam epitaxy of metamorphic $\operatorname{In}_{\mathrm{y}} \mathrm{Ga}_{1-\mathrm{y}} \mathrm{P}$ solar cells on mixed anion $\mathrm{GaAs}_{\mathrm{x}} \mathrm{P}_{1-\mathrm{x}} / \mathrm{GaAs}$ graded buffers" J. Vac. Sci. Technol. B 29, 03 C118 (2011)

${ }^{38}$ J.L. McCarter, M.L. Stutzman, K.W. Trantham, Timothy G. Anderson, A.M. Cook, and Timothy J. Gay, “A lowvoltage retarding-field Mott polarimeter for photocathode characterization” Nucl. Instr. and Meth. A 618,30 (2010)

${ }^{39}$ R. L. Bell, Negative electron affinity devices, (Clarendon, Oxford, England, 1973) 